\title{
Does Normal Breathing Rate Effects Interest of People in Cricket?
}

\author{
Muhammad Imran Qadir and Arslan Hassan* \\ Institute of Molecular Biology and Biotechnology, Pakistan
}

*Corresponding author: Arslan Hassan, Institute of Molecular Biology and

Biotechnology, Bahauddin Zakariya University, Pakistan.

Received Date: March 05, 2019

Published Date: March 20, 2019

\begin{abstract}
The intention of our current study was to relate the normal breathing rate of people with the interest of people in cricket [1]. The breathing rate or respiration rate is the rate at which a normal person breaths per minute. Normally the breathing rate is measured by respirometer when the person is in rest state [2]. The respiration rate may be increase due to illness or diseases [3]. A normal person breath in minute about 12-18 times. A normal rate can change with the increasing age or level of activity. Cricket is a professional game in the world and many people take interest in cricket and become fans of their favourite teams or become fans of just single player. There are many categories of fans that watch and play cricket as it gives them relaxation from their boring routine and keeps them mentally and physically fit.
\end{abstract}

Keywords: Cricket; Breathing rate; Stopwatch; Respirometer

\section{Introduction}

The breathing rate or respiration rate is the rate at which a normal person breaths per minute. Normally the breathing rate is measured by respirometer when the person is in rest state. The respiration rate may be increase due to illness or diseases. A normal person breath in minute about 12-18 times. Normal breathing rate change with the age differences such from the birth of baby to 7 weeks the breathing rate is 30-40 age up to 6 months breathing rate is 25 to 40 and at age of 6 breathing rate is $20-25$ and adults have normal breathing rate is 12 to 18 . Sometimes babies have the breathing rate more than 50 which means they are suffering from the serious diseases. The normal breathing rate is the indicator for respiratory diseases. There are many reasons that are responsible for fluctuation in the normal breathing rate of a person such as anxiety in which person breaths faster than the normal, in fever with the increase in the temperature of body the respiration rate also increases, respiratory illnesses such as asthma or pneumonia produces difficulties for breathing which can increase the breathing rate hearts diseases and dehydration both can increase the respiration rate of a person [4,5]. A normal rate can change with the increasing age or level of activity but when there is range of breathing is too much or too much low it means that this is a medical problem and call for doctor.
Cricket is a professional game in the world and many people take interest in cricket and become fans of their favorite teams or become fans of just single player [6]. There are many categories of fans that watch and play cricket as it gives them relaxation from their boring routine and keeps them mentally and physically fit. Some are fanatic types of fans of cricket which go in ground with full preparation as they are going on the fashion show and they can live or can die with the score at the end. Some fans are the talker which talks all the match and won't stop and they have no sports etiquette at all but gives us good company. Cricket have some loudmouth fans for which we cannot say anything whether they are lover or hater of cricket. Some are the fantasy team watcher which only talk about their favorites not for any other they do not focus on a single game. Some fans are the blind followers which are just spectator and mix up with those which cheers against their teams they just want to be always in action of cheering [7]. Some are the loyal season ticket holders which are the heart and souls of fans and they can't think of any other teams except their own team. They are sports lover.

\section{Material and Methods}

\section{Protocol to measure the respiration rate or breathing rate}

Firstly, we gained the subject's consent to measure the normal breathing rate. Then we sat them on the comfortable position and 
on the normal temperature so low temperature in surrounding could cause shivering which could increase the breathing rate. We allowed them to sit for almost 20 minutes for complete comfort for taking precise measurement of breathing rate. Then we started to measure the respiration rate the number of chest movement up and down with the time by using stopwatch holding in hand. Then we again questioned them that they were interested in cricket or not. They gave us answers and we listed them one by one with their normal breathing range [8]. Then we calculated the average normal breathing rate of all subjects and also their standard deviations. Total of 130 subjects take their part in current survey. All the subjects were university students.

\section{Statistical Analysis}

Statistical Analysis and T-test was made by using Microsoft Excel.

\section{Results and Discussion}

The Table 1 shows that males with the average normal breathing rate of 27 with SD of 3.06 are interested in cricket while the other with 24 average normal breathing rate and with SD of 2.23 are not interested in cricket and $p$ value is greater than the standard $p$ value which shows non-significant relation between both. Then females which have 26 average breathing rate or respiration rate with the SD of 2.91 are lovers of cricket and other with 25 and SD of 2.54 are not lovers of cricket [9]. Then the $p$ value which is higher than the normal which shown there is non-significant relation between interest of people in cricket with the normal average breathing rate of people [10].

Table 1: $p>0.1$ so the $p$ value shows non-significant relation between the normal breathing rate and interests of people in cricket.

\begin{tabular}{|c|c|c|c|}
\hline Gender & $\begin{array}{c}\text { People that have } \\
\text { Interest in Cricket }\end{array}$ & $\begin{array}{c}\text { People that have no } \\
\text { Interest in Cricket }\end{array}$ & P value \\
\hline Males & $27 \pm 3.06$ & $24 \pm 2.23$ & 0.6 \\
\hline Females & $26 \pm 2.91$ & $25 \pm 2.54$ & 0.5 \\
\hline
\end{tabular}

\section{Conclusion}

The conclusion from the current survey is that there is no scientific relation between interest of people in cricket with the normal respiration rate.

\section{Acknowledgement}

None.

\section{Conflict of Interest}

No conflict of interest.

\section{References}

1. Thakur A, Aggarwal P, Siddiqui U (2018) Analysis of Pulmonary Diseases Using Wireless Breathing Rate and Pulse Rate Monitoring System. In 2018 Second International Conference on Inventive Communication and Computational Technologies (ICICCT).

2. Porto AA, Tavares BS, Vidigal G, Garner DM, Raimundo RD, et al. (2018) Nonlinear Dynamics of Heart Rate During Slow Breathing Exercise. Indian J Physiol Pharmacol 62(2): 160-169.

3. Qadir MI, Javid A (2018) Awareness about Crohn's Disease in biotechnology students. GloAdv Res J Med Medical Sci 7(3): 062-064.

4. Qadir MI, Saleem A (2018) Awareness about ischemic heart disease in university biotechnology students. GloAdv Res J Med Medical Sci 7(3): 059-061.

5. Qadir MI, Ishfaq S (2018) Awareness about hypertension in biology students. Int J Mod Pharma Res 7(2): 08-10.

6. Qadir MI, Mehwish (2018) Awareness about psoriasis disease. Int J Mod Pharma Res 7(2): 17-18.

7. Qadir MI, Shahzad R (2018) Awareness about obesity in postgraduate students of biotechnology. Int J Mod Pharma Res 7(2): 14-16.

8. Qadir MI, Rizvi M (2018) Awareness about thalassemia in post graduate students. MOJ Lymphology \& Phlebology 2(1): 14-16.

9. Qadir MI, Ghalia BA (2018) Awareness survey about colorectal cancer in students of M. Phil Biotechnology at Bahauddin Zakariya University, Multan, Pakistan. Nov Appro in Can Study 1(3): NACS.000514.2018.

10. Qadir MI, Saba G (2018) Awareness about intestinal cancer in university student. Nov Appro in Can Study 1(3): NACS.000515.2018. 